\title{
Green's functions for polarized radiative transfer equation in different geometries
}

\author{
Juris Freimanis ${ }^{1,2}$ \\ ${ }^{1}$ Ventspils International Radio Astronomy Centre, Ventspils University College, \\ Inzenieru iela 101a, LV-3600 Ventspils, Latvia \\ email: jurisf@venta.lv \\ ${ }^{2}$ Institute of Mathematical Sciences and Information Technologies, Liepaja University, \\ Liela iela 14, LV-3401 Liepaja, Latvia
}

\begin{abstract}
A review of some earlier exact analytic solutions of monochromatic stationary vector radiative transfer equation in homogeneous infinite medium is given. It is stressed that Green's functions for plane-parallel, spherical and cylindrical symmetry are expressed through derivatives and integrals from basically one and the same set of functions.
\end{abstract}

Keywords. Polarization, radiative transfer, methods: analytical

\section{Introduction}

AGB stars, post-AGB objects, protoplanetary nebulae and young planetary nebulae often have gas-dust shells of complex morphology and nontrivial geometry. Radiation coming from these objects is often highly polarized.

Precise modeling of such objects necessarily includes numerical solution of polarized radiative transfer equation (RTE). Simultaneously it is desirable to obtain analytic solutions of RTE in all the simplest cases which allow to do it, at least in order to test the numerical methods. The analytic properties of the solutions should be studied and reproduced in the numerical schemes. Green's functions are of particular importance because they give the solution of RTE for arbitrary primary sources of radiation.

This contribution is a short review of the author's results in this field. Green's functions for homogeneous isotropic infinite medium with radiation field having plane-parallel, spherical or cylindrical symmetry have been found explicitly. Different integral representations of one and the same set of basis functions allow to describe the radiation field with different symmetries.

\section{The physical assumptions}

Let us consider statistically stationary, homogeneous and isotropic turbid medium in Euclidean space, without circular birefringence nor circular dichroism. The scattering process is monochromatic, with single scattering albedo $0<\lambda \leqslant 1$. The single scattering matrix is integrable in Lebesgue's sense on unit sphere, and physically valid according to Konovalov (1985) for almost all scattering angles $\Theta \in[0, \pi]$. The scattering medium is mirror symmetric according to Mishchenko et al. (2002). The radiation field is assumed to be stationary.

Let the single scattering matrix be sufficiently smooth in order to satisfy the analyticity conditions defined by Freimanis (1993), Eq. (6), or Freimanis (2005), Eq. (20). For each 
$s=0, \pm 1, \pm 2, \ldots$ the so-called dispersion matrix $\boldsymbol{\Lambda}_{s}(\eta)$ is assumed to be nonsingular on the sides of the cut $\eta \in[-1,1]$ drawn in the complex plane (see Freimanis (1993), Eq. 55, or Freimanis (2005), Eq. 23).

\section{Summary of the results}

Radiation field can be described with Stokes vector $\mathbf{I}(\mathbf{r}, \boldsymbol{\Omega})$, where $\mathbf{r}$ is the spatial radius-vector and $\boldsymbol{\Omega}$ is the direction of propagation of radiation. Polarized RTE in arbitrary coordinate system is as follows (Freimanis (2011)):

$$
\hat{\mathbf{L}} \mathbf{I}(\mathbf{r}, \boldsymbol{\Omega})=-\mathbf{I}(\mathbf{r}, \boldsymbol{\Omega})+\int_{4 \pi} \mathbf{R}(\chi) \boldsymbol{\Gamma}(\Theta) \mathbf{R}(\gamma) \mathbf{I}\left(\mathbf{r}^{\prime}, \boldsymbol{\Omega}^{\prime}\right) d \boldsymbol{\Omega}^{\prime}+\mathbf{B}_{0}(\mathbf{r}, \boldsymbol{\Omega}),
$$

where $\hat{\mathbf{L}}$ is a differential operator, and the subsequent terms in the r.h.s. describe extinction, scattering and primary sources. According to Morse \& Feshbach (1953), to find Green's function $\mathbf{G}\left(\mathbf{r}, \boldsymbol{\Omega} ; \mathbf{r}^{\prime}, \boldsymbol{\Omega}^{\prime}\right)$ for Eq. (3.1) means to find its general solution for arbitrary primary sources $\mathbf{B}_{0}(\mathbf{r}, \boldsymbol{\Omega})$, provided the solution exists and is unique:

$$
\mathbf{I}(\mathbf{r}, \boldsymbol{\Omega})=\int d \mathbf{r}^{\prime} \int_{4 \pi} \mathbf{G}\left(\mathbf{r}, \boldsymbol{\Omega} ; \mathbf{r}^{\prime}, \boldsymbol{\Omega}^{\prime}\right) \mathbf{B}_{0}\left(\mathbf{r}^{\prime}, \boldsymbol{\Omega}^{\prime}\right) d \boldsymbol{\Omega}^{\prime}
$$

With different physical assumptions, the expressions for Green's function with planeparallel symmetry were found by several authors. On conditions stated above, and using the apparatus of singular eigensolutions introduced by Case \& Zweifel (1967) and Domke (1975), this was done in Freimanis (1990) (infinite medium, plane-parallel symmetry of the radiation field), Freimanis (1993) (half-space), Freimanis (2005) (infinite medium, spherically symmetric radiation field), and Freimanis (2009) (infinite medium, cylindrically symmetric radiation field).

The resulting expressions are based on one and the same set of basis functions. In case of nonplanar geometries, the final formulae are quite long. The reader is referred to the original papers.

\section{Acknowledgements}

The participation of the author at the Symposium was financed from the project SATTEH (2010/0189/2DP/2.1.1.2.0/10/APIA/VIAA/019) being implemented in Ventspils University College.

\section{References}

Case, K. M. \& Zweifel, P. F. 1967, Linear Transport Theory. (Massachusetts: Addison-Wesley Publishing Company)

Domke, H. 1975, Journal of Quantitative Spectroscopy and Radiative Transfer, 15, 669

Freimanis, J. 1990, Investigations of the Sun and Red Stars, 32, 20

Freimanis, J. 1993, Investigations of the Sun and Red Stars, 36, 18

Freimanis, J. 2005, Journal of Quantitative Spectroscopy and Radiative Transfer, 96, 451

Freimanis, J. 2009, Journal of Quantitative Spectroscopy and Radiative Transfer, 110, 1307

Freimanis, J. 2011, Journal of Quantitative Spectroscopy and Radiative Transfer, 112, 2134

Konovalov, N. V. 1985, Polarization matrices corresponding to transformations within Stokes cone. (Preprint No. 171, Moscow: Institute of Applied Mathematics of the USSR Academy of Sciences)

Mishchenko, M. I., Travis, L. D., \& Lacis, A. A. 2002, Scattering, Absorption, and Emission of Light by Small Particles. (Cambridge et al.: Cambridge University Press)

Morse, P. M. \& Feshbach, H. 1953, Methods of Theoretical Physics. (New York: McGraw-Hill) 